\title{
An investigation into the use of CALNN capped gold nanoparticles for improving microwave heating.
}

\author{
Julian Bonello \\ Department of Physics \\ University of Malta \\ Msida, Malta \\ julian.bonello@um.edu.mt \\ Iman Farhat \\ Department of Physics \\ University of Malta \\ Msida, Malta \\ eman.farhat.10@um.edu.mt
}

\author{
Francesco Rossi \\ Department of Physics and Astronomy \\ University College London \\ London, England \\ francesco.rossi.15@ucl.ac.uk \\ Lourdes Farrugia \\ Department of Physics \\ University of Malta \\ Msida, Malta \\ lourdes.farrugia@um.edu.mt
}

\author{
Nguyen Thi Kim Thanh \\ Department of Physics and Astronomy \\ University College London \\ London, England \\ ntk.thanh@ucl.ac.uk \\ Charles V Sammut \\ Department of Physics \\ University of Malta \\ Msida, Malta \\ charles.v.sammut@um.edu.mt
}

\begin{abstract}
The use of microwaves for both therapeutic and diagnostic applications has become an accepted alternative in the clinics. For diagnostics, gold (Au) nanoparticles have been used for imaging tumour vasculature and also served as potential diagnostic markers for cancer. [1] In high-frequency therapeutic applications, two different treatments exist; hyperthermia and ablation. [2] In hyperthermia, the tumour tissue is heated to supra-physiological temperatures, making it more susceptible to traditional treatment methods such as chemotherapy and radiotherapy. This type of treatment could be administered externally. However, there still remains a challenge to focus the heating to particular areas which need to be treated, while avoiding unwanted hotspots. To date, numerous methods have been used to focus the heat from different antennas. A novel technique which is being investigated is the use of nanoparticles to improve focusing and thus achieve better localised heating effect. A previous study by Cardinal et al. [3] showed that at RF-frequencies, remarkable improvements resulted from using Au nanoparticles.
\end{abstract}

In this work, the use of CALNN peptide capped Au nanoparticles for the focusing of microwaves at $2.45 \mathrm{GHz}$ is investigated. The CALNN capped Au nanoparticles were prepared as described elsewhere. [4] Au nanoparticles were added to tissue mimicking solutions (such as muscle, liver or fat) to compare their dielectric properties with the those of the control (without $\mathrm{Au}$ nanoparticles). The frequency range investigated was from $400 \mathrm{MHz}$ to $20 \mathrm{GHz}$. During this study, various concentrations, particle sizes and shapes of $\mathrm{Au}$ nanoparticles were considered. The study also investigated the heating rates of the pseudo-biological tissue samples and how these varied with the addition of the nanoparticles. The outcome of this study will determine the viability of using CALNN capped Au nanoparticles to assist in the focusing of microwave radiation during microwave hyperthermia.

Keywords- Dielectric Properties, Au Nanoparticles, Microwave Hyperthermia

\section{INTRODUCTION}

The use of microwaves in the medical industry for thermal therapies has become wildly accepted. Some of the medical conditions treated with microwaves are cardiac arrhythmias, Parkinson's disease, hyperopia and cancer. There are two categories of thermal treatment; ablative or hyperthermic procedures. The latter procedures involve warming specific tissue to between $42^{\circ}$ and $45^{\circ} \mathrm{C}$ [2] by the external administration of RF or microwave fields, focussed on the treatment region while avoiding unwanted hotspots.

Gold Nanoparticles (GNPs) have been shown to have an excellent uptake by mammalian cancerous cells [5] and are also known to have low toxicity [6]. These two factors make them a good candidate to be used for microwave-assisted cancer therapies. To date, GNPs have been utilised in cancer diagnosis, treatment and even for drug delivery [7]. When targeting cancer cells, the GNPs are normally attached to carbohydrates, antibodies and pharmacological agents, achieved through established conjugation techniques.

In most cases, solid-organ malignancies are treated by surgical resection, radiotherapy or chemotherapy, or a combination of these. Microwave hyperthermia is known to improve the effectiveness of chemotherapy and radio therapy. In a study performed by Hahn et al. [8] it is reported that while heating normal tissue to $41{ }^{\circ} \mathrm{C}$ would improve the rate at which cells are killed, further increasing the tumour temperature to $43{ }^{\circ} \mathrm{C}$ improves the killing rate significantly. There have therefore been numerous efforts to increase the tumour tissue without significantly altering the temperature of the surrounding tissue and having unwanted hotspots. Two examples of these efforts were those of Johnson [9] and Hergt [10], who used magnetic nanoparticles to focus microwaves.

The investigation of dielectric properties of biological samples is an established method of explaining how electromagnetic waves interact with biological tissue [11-14]. Few studies investigated the use of GNPs for microwave hyperthermia and to the authors' knowledge, none have investigated the effect of GNPs on the dielectric properties.

In this paper, the use of CALNN capped GNPs is investigated for the purpose of focusing the incident beam. This investigation focuses on measuring the dielectric properties of liver tissue mimics and how these alter when GNPs are added. 


\section{METHOD}

\section{A. GNPs preperation}

The preparation of the GNPs was done as described in [4]. Once the GNPs were prepared, the particle size was determined both using Dynamic Light Scattering (DLS) and UV-Visible spectrophotometry. Furthermore, the solution was also examined using Transmission Electron Microscopy (TEM). These images showed that the resultant GNPs did not aggregate. The size of the GNPs used in this investigation was $21 \mathrm{~nm}$.

\section{B. Tissue Mimic preperation}

Relevant to the effective medium theory, a mixing rule was employed to model the dielectric properties of the tissue under test. This method has resulted in accurate mimicking solutions of in vivo and ex vivo tissue in different states of hydration [15]. Assuming the tissues as a two-phase mixture, the models were generated considering a dry inclusion phase and a fluid-based host environment. The fluid-based environment was synthesized as a physiological solution by dissolving phosphate buffered saline (PBS) in deionized water. The dry inclusion phase was considered as protein (albumin), the dominant constituent of liver, and incorporated as a fraction corresponding to their prevalence in the tissue. Mixture solutions of PBS containing concentrations of bovine serum albumin (BSA), were prepared with the aim of accurately mimicking the biochemical composition of porcine liver which has a concentration of approximately $40 \%$ protein in an isotonic saline solution. Once the mimic was prepared it was mixed with the GNPs. The concentration of GNPs was calculated using the method explained in [17].

\section{Dielectric measurment procedure}

The dielectric measurements were conducted using a Rhode and Schwarz Vector Network Analyser (VNA) ZVA-50 and reflection measurements were performed using the Slim Form probe (Keysight N1501A). The frequency range investigated was from 0.5 to $50 \mathrm{GHz}$, with 501 points. The conversion from S11 parameters to the complex permittivity was done using the Keysight software $85070 \mathrm{E}$. The temperature of the samples being investigated was measured using a digital thermometer (DTM-3000) and measurements were done prior and post dielectric measurements to monitor any fluctuation in temperature.

Prior to conducting dielectric measurements on the tissue mimicking solutions the VNA was calibrated using a standard single port calibration with an open, short and load. The open required the probe to radiate into free space, the short required the probe to be shorted at its end while the load required the open end of the probe to be immersed into deionised water. Following the calibration, a validation of the measurement setup was performed using $0.1 \mathrm{~N}$ Sodium Chloride $(\mathrm{NaCl})$ solution. The measured values were compared to theoretical values for this solution.

Prior to the addition of GNPs, the dielectric measurements of the liver mimic solution were performed. The GNPs were then added to the mimicking solutions and shaken using a vortex mixer. The concentration of GNPs in the liver tissue mimic at this stage was $5.85 \mathrm{nM}$. The dielectric measurements were once again recorded with the first concentration of GNPs in the mixture. A second dose of GNPs was added to the liver mimicking solution and the dielectric properties were once again recorded. When the second batch of GNPs were added the concentration was $11.70 \mathrm{nM}$. The temperature of the tissue mimicking solution under investigation was monitored throughout the dielectric measurements and was $23{ }^{\circ} \mathrm{C}$.

\section{RESULTS}

Figures 1 and 2 present the real and imaginary parts of the relative permittivity as functions of frequency. Each trace in these plots depicts a different concentration of GNPs. The blue line represents the tissue mimic without any GNPs, the orange is a representation of the first dilution of GNPs in tissue mimic, while the grey line shows the results of the second concentration of GNPs in the mimic.

Figure 1 shows that as the GNPs are added, the real part of the permittivity decreases throughout the frequency spectrum investigated. This does not change at low frequency even when the concentration is doubled. Above $30 \mathrm{GHz}$ the variation in the real permittivity decreases minimally when doubling the concentration of GNPs. This result is also shown in Figure 3, where the percentage difference in the real part of the permittivity for the different concentrations is plotted as a function of frequency. The variations in the real part of the permittivity are within measurement uncertainty, which throughout the frequency range investigated were a maximum of $5.7 \%$.

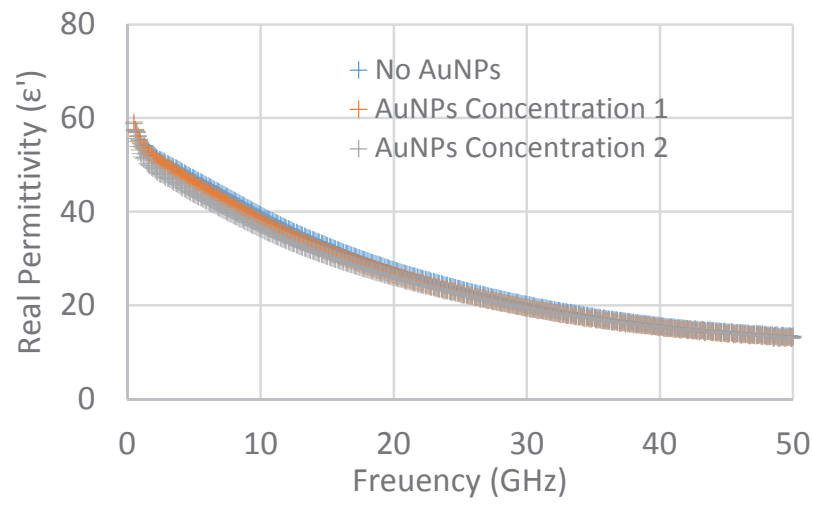

Figure 1. The measured real part of the permittivity of the tissue mimic solution and tissue mimic with different concentrations of GNPs.

Figure 2 shows how the imaginary part of the permittivity varies as GNPs are added. This shows that at low frequencies there is a significant increase in the imaginary part. This positive change decreases with increasing frequency. Above 9.2 $\mathrm{GHz}$ the addition of GNPs causes a decrease in the measured imaginary part of the permittivity. This variation is also shown in Figure 4, where the calculated percentage difference between the liver mimic without GNPs and that with GNPs is at a maximum of $43.94 \%$. The fact that at 2.45 $\mathrm{GHz}$ there is an increase in the imaginary part is a desirable effect as this would mean that there will also be an increase in the specific absorption rate of the tissue, and hence in the 
heat dissipated. The maximum measurement uncertainty in the imaginary part of the permittivity was $4.9 \%$.

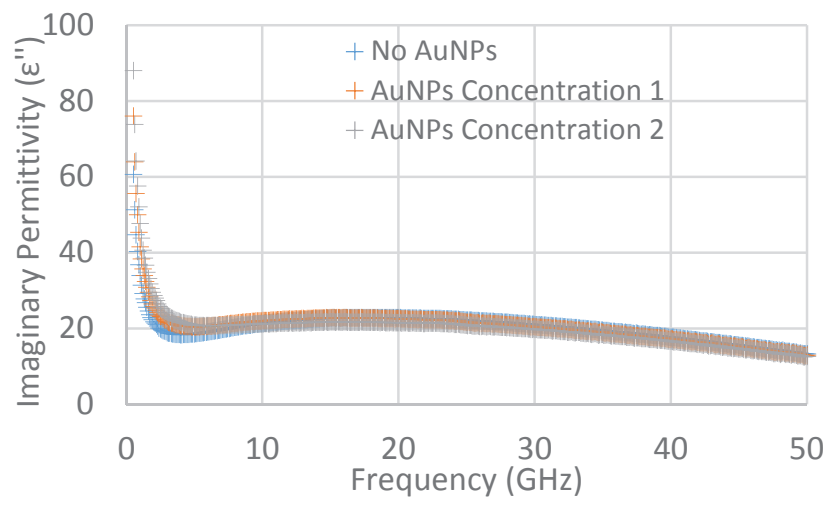

Figure 2. The measured imaginary part of the permittivity of the tissue mimic solution and tissue mimic with different concentrations of GNPs.

The samples under investigation were also heated in a microwave oven simultaneously and their temperature was monitored. This resulted in a temperature increase of $45.8^{\circ} \mathrm{C}$ in the tissue mimic with GNPs while a temperature of $43{ }^{\circ} \mathrm{C}$ in the tissue mimic by its own, over the same time interval and with the same power setting.

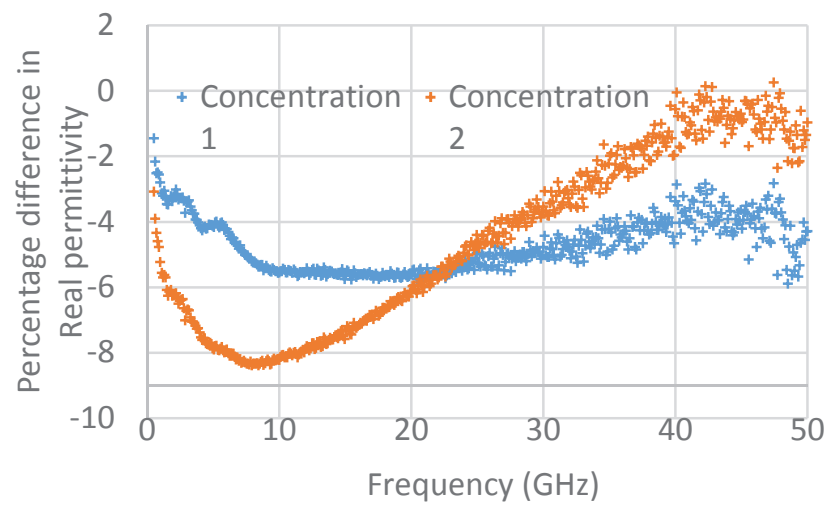

Figure 3. The percentage difference in the real part of the permittivity as a function of frequency at different GNP concentrations (Concentration $1=5.85 \mathrm{nM}$ and Concentration 2= $11.70 \mathrm{nM})$.

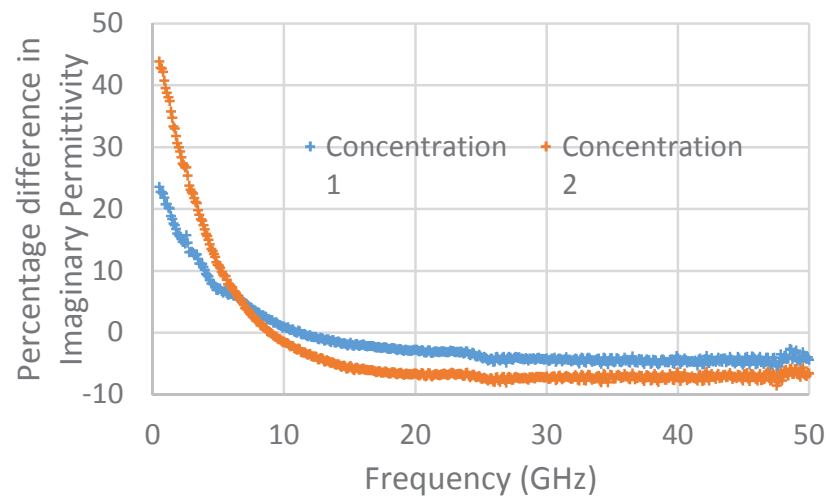

Figure 4. The percentage difference in the imaginary part of the permittivity as a function of frequency at different GNP concentrations (Concentration $1=5.85 \mathrm{nM}$ and Concentration 2= $11.70 \mathrm{nM})$.

\section{CONCLUSIONS}

This study investigates the potential for the use of GNPs for the focusing of microwaves that would be useful for microwave hyperthermic applications. The preliminary results obtained in this paper show that there is potential for the use of GNPs for the focusing of microwaves in hyperthermic applications. This results from a significant increase in the imaginary part of the permittivity with the addition of GNPs, and the consequent localized increase of the associated SAR. The conclusions reached will serve as ground work for further studies where the size and shape of the GNPs will be investigated further in respect of their effectiveness in focusing microwaves and achieving preferential heating of targeted tissue during microwave hyperthermia.

\section{REFERENCES}

[1] Cai, W., Gao, T., Hong, H. and Sun, J., 2008. Applications of gold nanoparticles in cancer nanotechnology. Nanotechnology, science and applications, 1, p.17-32

[2] Rossmann, C. and Haemmerich, D., 2014. Review of temperature dependence of thermal properties, dielectric properties, and perfusion of biological tissues at hyperthermic and ablation temperatures. Critical Reviews ${ }^{\mathrm{TM}}$ in Biomedical Engineering, 42(6), p. 467-492

[3] Cardinal, J., Klune, J.R., Chory, E., Jeyabalan, G., Kanzius, J.S., Nalesnik, M. and Geller, D.A., 2008. Noninvasive radiofrequency ablation of cancer targeted by gold nanoparticles. Surgery, 144(2), p.125-132.

[4] Lévy, R., Thanh, N.T., Doty, R.C., Hussain, I., Nichols, R.J., Schiffrin, D.J., Brust, M. and Fernig, D.G., 2004. Rational and combinatorial design of peptide capping ligands for gold nanoparticles. , 126(32), p.10076-10084.

[5] Chithrani, B.D., Ghazani, A.A. and Chan, W.C., 2006. Determining the size and shape of gold nanoparticle uptake into mammalian cells. Nano letters, 6(4), p.662-668.

[6] Connor, E.E., Mwamuka, J., Gole, A., Murphy, C.J. and Wyatt, M.D.,

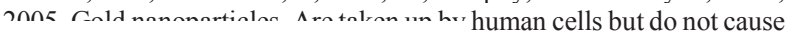
acute cytotoxcity. Small, 1(3), p.325-327.

[7] Singh, P., Pandit, S., Mokkapati, V.R.S.S., Garg, A., Ravikumar, V. and Miiakovic. I.. 2018. Gold nanoparticles in diagnostics and therapeutics for human cancer. International journal of molecular sciences, 19(7), p.1979.

[8] Hahn, G.M., Braun, J. and Har-Kedar, I., 1975. Thermochemotherapy: synergism between hyperthermia (42-43 degrees) and adriamycin (of bleomycin) in mammalian cell inactivation. Proceedings of the National Academy of Sciences, 72(3), p.937-940.

[9] Johannsen, M., Gneveckow, U., Eckelt, L., Feussner, A., Waldöfner, N., Scholz, R., Deger, S., Wust, P., Loening, S.A. and Jordan, A., 2005. Clinical hyperthermia of prostate cancer using magnetic nanoparticles: presentation of a new interstitial technique. International journal of hyperthermia, 21(7), pp.637-647.

[10] Hergt, R., Dutz, S., Müller, R. and Zeisberger, M., 2006. Magneitc particle hyperhtermia: nanoparticle magnetism and materials development for cancer therapy. Journal of Physics: Condesed Matter, 18(38), p.S2919-S2934

[11] Bonello, J., Elahi, M.A., Porter, E., O'Halloran, M., Farrugia, L. and Sammut, C.V., 2018. An investigation of the variation of dielectric properties of ovine lung tissue with temperature. Biomedical Physics and Engineering Express.

[12] Gabriel, C. and Peyman, A., 2018. Dielectric properties of biological tissues; variation with age. In Conn's Handbook of Models for Human Aging (Second Edition),p. 939-952.

[13] Lazebnik, M., Popovic, D., McCartney, L., Watkins, C.B., Lindstrom, M.J., Harter, J., Sewall, S., Ogilvie, T., Magliocco, A., Breslin, T.M. and Temple, W., 2007. A large-scale study of the ultrawideband microwave dielectric properties of normal, benign and malignant breast 
tissues obtained from cancer surgeries. Physics in Medicine and Biology, 52(20), p.6093-6115.

[14] Farina, L., Pinto, R., Lopresto, V., Ruvio, G., Vannucci, L. and Cavagnaro, M., 2018, September. Ex vivo dielectric properties of fat: influence of the experimental conditions on the measured data. In 2018 EMF-Med 1st World Conference on Biomedical Applications of Electromagnetic Fields (EMF-Med), p.1-2. IEEE.

[15] Pollacco, D.A., Farrugia, L., Conti, M.C., Farina, L., Wismayer, P.S. and Sammut, C.V., 2019. Characterization of the dielectric properties of biological tissues using mixture equations and correlations to different states of hydration. Biomedical Physics and Engineering Express.
[16] Haiss, W., Thanh, N.T., Aveyard, J. and Fernig, D.G., 2007. Determination of size and concentration of gold nanoparticles from UV-Vis spectra. Analytical chemistry, 79(11), p.4215-4221.

\section{ACKNOWLEDGMENT}

This work is supported by the MyWAVE COST Action CA17115 which funded a Short-Term Scientific Mission (STSM). 